\title{
Determination of light and strange quark condensates
}

\section{Craig McNeile ${ }^{* \dagger}$}

Bergische Universität Wuppertal,

E-mail: craig.mcneile@plymouth.ac.uk

I review the first determintion of the strange and light quark condensates in full lattice QCD. This was done by direct calculation of the expectation value of the trace of the quark propagator followed by subtraction of the appropriate perturbative contribution to convert to a value for the condensate in the MS-bar scheme at $2 \mathrm{GeV}$. Lattice QCD configurations including u, d, s and c quarks in the sea with $\mathrm{u} / \mathrm{d}$ quark masses going down to the physical value were used. The ratio of the strange to the light quark condensate was found to be 1.08(16).

31 st International Symposium on Lattice Field Theory LATTICE 2013

July 29 âĂS August 3, 2013

Mainz, Germany

${ }^{*}$ Speaker.

${ }^{\dagger}$ Supported by SFB-TR 55. 


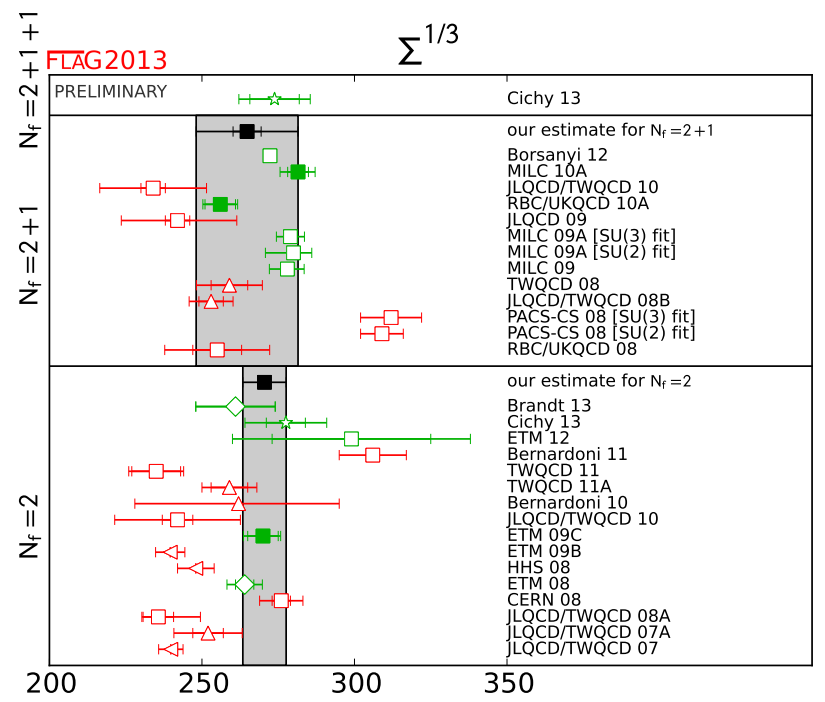

Figure 1: Review of results for the chiral condensate from the FLAG 2013 review [1].

\section{Introduction}

A critical feature of the nonperturbative dynamics of QCD at zero temperature is the condensation of quark-anti-quark pairs in the vacuum, spontaneously breaking the chiral symmetry of the action. The value of the chiral condensate (the quark condensate at zero quark mass) is then an important parameter for low energy QCD [2]. The well-known Gell-Mann, Oakes, Renner (GMOR) relation [3]:

$$
\frac{f_{\pi}^{2} M_{\pi}^{2}}{4}=-\frac{m_{u}+m_{d}}{2} \frac{\langle 0|\bar{u} u+\bar{d} d| 0\rangle}{2}
$$

connects the $u / d$ quark masses times condensate to the square of the mass times decay constant for the Goldstone boson of the spontaneously broken symmetry. Eq.( 1.1) has normalisation such that $f_{\pi}=130 \mathrm{MeV}$. The GMOR relation holds in the limit of $m_{u}, m_{d} \rightarrow 0$. A value for this chiral condensate can be derived from the chiral extrapolation of lattice QCD results for light meson masses and decay constants. Non-perturbative methods have been developed to compute the QCD condensate. The Flavor Lattice Averaging Group (FLAG [1]) have recently reviewed the lattice QCD calculations $[4,5,6]$ of the chiral condensate. Figure 1 is the summary plot for $\Sigma^{1 / 3}(2$ $\mathrm{GeV}$ ) (the chiral condensate) from the 2013 update of the FLAG review [7]. FLAG quote as their averaged value 265 (17) $\mathrm{MeV}$ for the chiral condensate in the $\overline{M S}$ scheme at $2 \mathrm{GeV}$ with $n_{f}=2+1$ sea quarks.

The determination of the quark condensate for non-zero quark masses is more problematic because, depending on the method used, there are various sources of unphysical quark mass dependence and a careful definition of the condensate is required. This definition must be phrased in terms of the Operator Product Expansion (OPE) since this is the context in which the condensate appears $[8,2,9]$. The OPE allows a separation of short and long-distance contributions in, for example, a short-distance current-current correlator. The expansion is in terms of a set of matrix 


\begin{tabular}{|c|c|c|}
\hline Group & application & $\frac{\langle\bar{s} s\rangle}{\langle\overline{l l}\rangle}$ \\
\hline JLQCD [12] & $\alpha_{s}$ & 1 \\
HPQCD [13] & strange-heavy moments & 0.7 \\
Borsanyi et al. [14] & hadron resonance gas & 0.8 \\
\hline
\end{tabular}

Table 1: Some values for $\frac{\langle\bar{s} s\rangle}{\langle\bar{l}\rangle\rangle}$ used in lattice QCD calculations.

elements of local operators multiplied by coefficient functions. The aim is for all the long-distance contributions (with scale $<\mu$ ) to be contained in the matrix elements and the short distance contributions (with scale $>\mu$ ) in the coefficient functions. A key matrix element, since it corresponds to a relatively low-dimensional $(d=3)$ operator, is that of the quark condensate. The clean separation of scales in the OPE only works if the local operators are not normal ordered [10,11]. Then the coefficient functions are analytic in the quark masses and therefore free of infrared sensitivity. This means, however, that the quark mass dependent mixing of $m \bar{\psi} \psi$ with the unit operator must be taken into account and that the vacuum matrix element of $m \bar{\psi} \psi$ is not cut-off independent. The quantity that appears in the OPE is the vacuum matrix element in, for example, the $\overline{M S}$ scheme at the scale $\mu$. This matrix element can be derived from lattice QCD and I quote results here for $\mu=$ $2 \mathrm{GeV}$. The results can easily be run to other scales, as appropriate.

The value of the condensate for quarks of non-zero mass up to that of the strange quark is needed in a number of calculations involving light quark correlators. Table 1 contains some values for $\frac{\langle\bar{s} s\rangle}{\langle\bar{l}\rangle\rangle}$ used in lattice QCD calculations, or models used to analyse the results of lattice QCD calculations. A continuum example where the strange quark condensate is needed is in the determination of the strange quark mass, $m_{s}$, from hadronic $\tau$ decays [15].

Current estimates of the value of the strange quark condensate vary by almost a factor of two $[16,17]$. It is not even clear whether the strange condensate is larger or smaller than the light quark condensate. For very large quark masses, $m_{q}>\Lambda_{Q C D}$, say, so that the quark mass dominates the propagator, it seems clear that the condensate should fall to zero, but this does not help in determining the slope of the condensate with $m_{q}$ for small quark masses.

In this paper I review the determination of the strange (or other non-zero mass) quark condensate by direct calculation in full lattice QCD. There has been essentially no previous work on computing the mass dependence of the quark condensates using lattice QCD. The talk was a based on the paper [18] where full details can be be found.

The strange quark and light quark propagators were directly determined on a range of gluon field configurations at different values of the lattice spacing and sea quark masses. To isolate the low-energy nonperturbative value of the condensate from these results requires the subtraction of a perturbative contribution. The perturbative contribution in lattice QCD has two pieces. One diverges as $a \rightarrow 0$ and dominates the vacuum expectation value of the strange quark propagator, particularly on the finer lattices. The second piece contains infrared sensitive logarithms of the quark mass which cancel against similar terms in continuum perturbation theory allowing an infrared safe definition of the condensate for use in the OPE, as discussed above.

The error in the final result then depends on how well this subtraction can be done. An explicit calculation of the perturbative pieces through $\mathscr{O}\left(\alpha_{s}\right)$ and fit for unknown higher order terms was 
used. The known quark mass and $a$ dependence of these unknown terms helps in constraining them along with the very small statistical errors in the lattice results. A particularly good discretisation of the Dirac action known as the Highly Improved Staggered Quark (HISQ) formalism [19] was used in 'second generation' gluon field configurations so that discretisation errors in the physical nonperturbative results are small.

\section{The problem of power divergences}

The direct determination of the chiral condensate in lattice QCD requires the calculation of the expectation value over an ensemble of gluon fields, $U$, of $\operatorname{Tr} M^{-1}$ where $M$ is the lattice discretisation of the Dirac matrix. The quark action for a given quark flavor,

$$
S_{f}=\bar{\psi} M_{f} \psi
$$

and

$$
\langle\bar{\psi} \psi\rangle=\left\langle 0\left|\bar{\psi}_{f} \psi_{f}\right| 0\right\rangle=-\frac{1}{V}\left\langle\operatorname{Tr} M_{f}(U)^{-1}\right\rangle_{U}
$$

where the trace is over spin, colour and space-time point and the gluon fields in the ensemble used for the average include the effect of sea quarks (of all flavors, not just $f$ ) in their probability distribution. $V$ is the lattice volume, $L^{3} \times T$. For a naive discretisation of the Dirac action $M$ takes the form:

$$
M=\gamma_{\mu} \Delta_{\mu}+m
$$

where $\Delta_{\mu}$ is a covariant finite difference on the lattice:

The difficulties in computing the mass dependence of the condensate can be seen from the leading order in continuum perturbation theory.

$$
\begin{aligned}
-\langle\bar{\psi} \psi\rangle & =\int_{0}^{\Lambda} \frac{d^{4} k}{(2 \pi)^{4}} \frac{12 m}{k^{2}+m^{2}} \\
& =\frac{3}{4 \pi^{2}}\left(m \Lambda^{2}+m^{3} \log \frac{m^{2}}{\Lambda^{2}+m^{2}}\right)
\end{aligned}
$$

Eq. 2.4 is the one loop continuum expression for the quark condensate for a massive quark. The $m \Lambda^{2}$ divergence in equation 2.4 depends on the regulator used. For example it is zero in $\overline{M S}$. On the lattice it is expected that a term of the form $m \Lambda^{2}$ will diverge with two powers of the inverse lattice spacing. This is also suggestive that the divergences $\frac{1}{a^{2}}$ will not be removed by using a fermion action, such as one obeying the Ginsparg-Wilson relation. Note however, there are new ideas on removing this type of divergence using the quark flow [20, 21]. There are additional potential divergences with Wilson like fermions. (see for example Hamber and David [22]).

The $m^{3} \log (m / \Lambda)$ term in Eq. 2.4 is universal since it arises from infrared part of the integral. The basic idea of our calculation is to subtract $\frac{1}{a^{2}}$ using perturbation theory. Additional constraints from taking the continuum limit were found. 


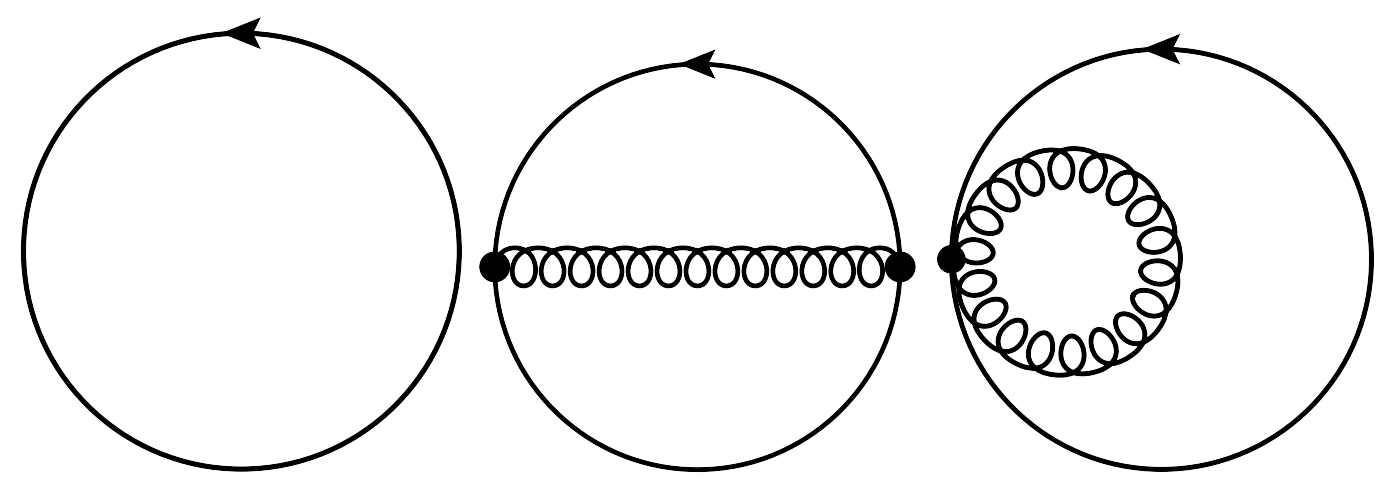

Figure 2: Perturbative contribution to the quark condensate through $\mathscr{O}\left(\alpha_{s}\right)$.

\section{Formalism for the calculation}

The lattice QCD calculation used the Highly Improved Staggered Quark (HISQ) action [19]. The graphs required to compute the quark condensate through $O\left(\alpha_{s}\right)$ for the HISQ action are in figure 2, and the results are Eq. 3.1 and figure 3.

$$
-a^{3}\langle\bar{\psi} \psi\rangle_{\mathrm{PT}, \mathrm{HISQ}}=a m_{0}\left[c_{0}\left(a m_{0}\right)+c_{1}\left(a m_{0}\right) \alpha_{s}+O\left(\alpha_{s}^{2}\right)\right]
$$
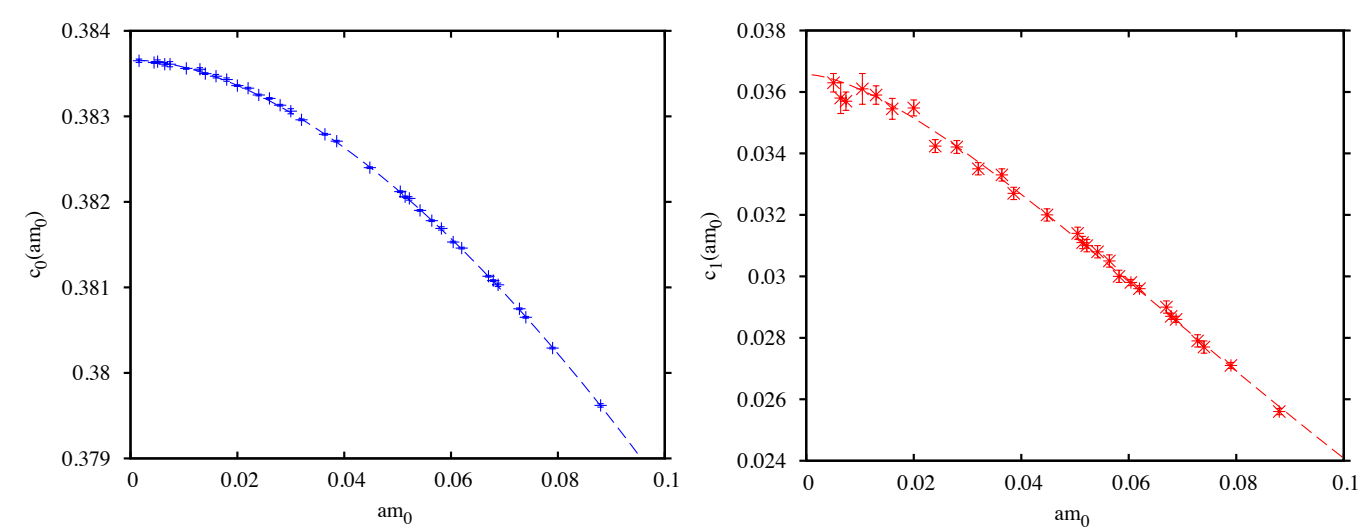

Figure 3: Zeroth- and first-order coefficients, $c_{0}$ and $c_{1}$ respectively, for the perturbative condensate, Eq. (3.1), versus the bare quark mass parameter $a m_{0}$ in lattice units. The uncertainties in $c_{0}$ resulting from numerical evaluations of the lattice loop integral are not visible in that plot. The fits given in the text are plotted as dashed lines.

The coefficients $c_{0}\left(a m_{0}\right)$ and $c_{1}\left(a m_{0}\right)$ were parametrised below. This also makes the log structure of the graphs explicit.

$$
\begin{gathered}
c_{0}\left(a m_{0}\right)=c_{00}+\left(a m_{0}\right)^{2}\left[c_{01} \log \left(a m_{0}\right)+c_{02}\right] \\
c_{1}\left(a m_{0}\right)=c_{10}+\left(a m_{0}\right)^{2}\left[c_{11} \log ^{2}\left(a m_{0}\right)+c_{12} \log \left(a m_{0}\right)+c_{13}\right]
\end{gathered}
$$

The condensate of strange or light quark extracted from the lattice needs to be compared against the condensates used in sum rule calculations. 
The extraction used a ratio of lattice to continuum perturbative factors (see appendix of [18] for some formalism). This also means that any issues with "renormalons" should cancel.

The one loop expression for the quark condensate in the $\overline{M S}$ scheme is:

$$
-\langle\bar{\psi} \psi\rangle_{\mathrm{PT}, \overline{\mathrm{MS}}}^{(\mu)}=\bar{m}^{3}(\mu) \times\left[d_{01} l_{m}+d_{02}+\alpha_{s}\left(d_{11} l_{m}^{2}+d_{12} l_{m}+d_{13}\right)+\ldots\right]
$$

where $l_{m}=\log (\bar{m}(\mu) / \mu)$.

To extract a massive condensate a perturbative subtraction term of the form

$$
\begin{aligned}
\Delta_{\mathrm{PT}} & =-a^{4}\left(\left\langle m_{0} \bar{\psi} \psi\right\rangle_{\mathrm{PT}, \mathrm{HISQ}}-\langle\bar{m}(\mu) \bar{\psi} \psi\rangle_{\mathrm{PT}, \overline{\mathrm{MS}}}\right) \\
& =c_{00}\left(a m_{0}\right)^{2}+\alpha_{s} c_{10}\left(a m_{0}\right)^{2}+\left(a m_{0}\right)^{4}\left[c_{01} l_{\mu}-0.077(1)\right] \\
& +\alpha_{s}\left(a m_{0}\right)^{4}\left[c_{11} l_{\mu}^{2}+0.1340(2) l_{\mu}+0.406(15)\right]+\ldots,
\end{aligned}
$$

was defined, where $l_{\mu}=\log (\mu a)$.

Of particular note is that the $\operatorname{logs}$ in Eq. 3.5 are of the form $\log (\mu a)$. Potential problems from $\log (m)$ terms in the perturbative expressions of the condensate for the HISQ action and $\overline{M S}$ cancel in Eq. 3.5. There is theoretical justification for form in Eq. 3.5 from the OPE in the appendix of [18].

The physical $\overline{M S}$ condensate at the scale $\mu$ :

$$
\langle m \bar{\psi} \psi\rangle_{N P, \overline{M S}}(\mu)=a^{-4}\left(a^{4}\langle m \bar{\psi} \psi\rangle_{0}-\Delta_{P T}\right),
$$

The cancellation of the $\log (m)$ terms in Eq. 3.5 only worked, because the perturbation theory was not normal ordered. One consequence of this is that $m_{q}\left\langle\overline{\psi_{q}} \psi_{q}(\mu)\right\rangle$ depends weakly on the renormalisation scale. Most modern sum rule calculations of the condensate also don't use normal ordered perturbation theory.

\section{Lattice QCD calculation on $2+1+1$ gluon configurations}

The main calculation used gauge configurations with $2+1+1$ flavours of HISQ sea quarks. These were 2nd generation configurations generated by the MILC collaboration [23]. Ensembles with three lattice spacings: $0.15,0.12$ and $0.09 \mathrm{fm}$, and pion masses at the physical point, were used in the analysis (see [18] for more details).

The following relation was used to compute the bare condensate.

$$
-a^{3}\langle\bar{\psi} \psi\rangle_{0}=\left(a m_{q}\right) \sum_{t} C_{\pi}(t)
$$

where $C_{\pi}(t)$ is the correlator for the Goldstone pion. There is a diagrammatic proof in ref. [24] (see also the discussion in our paper [18]). It is also easy to compute $-a^{3}\langle\bar{\psi} \psi\rangle_{0}$ using noise sources, but Eq. 4.1 can be used with correlators generated for to measure the mass of the pion.

In the analysis $R_{l}$ is introduced

$$
R_{l}=-\frac{4 m_{l}\left\langle\bar{\psi} \psi_{l}\right\rangle}{\left(f_{\pi}^{2} M_{\pi}^{2}\right)}
$$




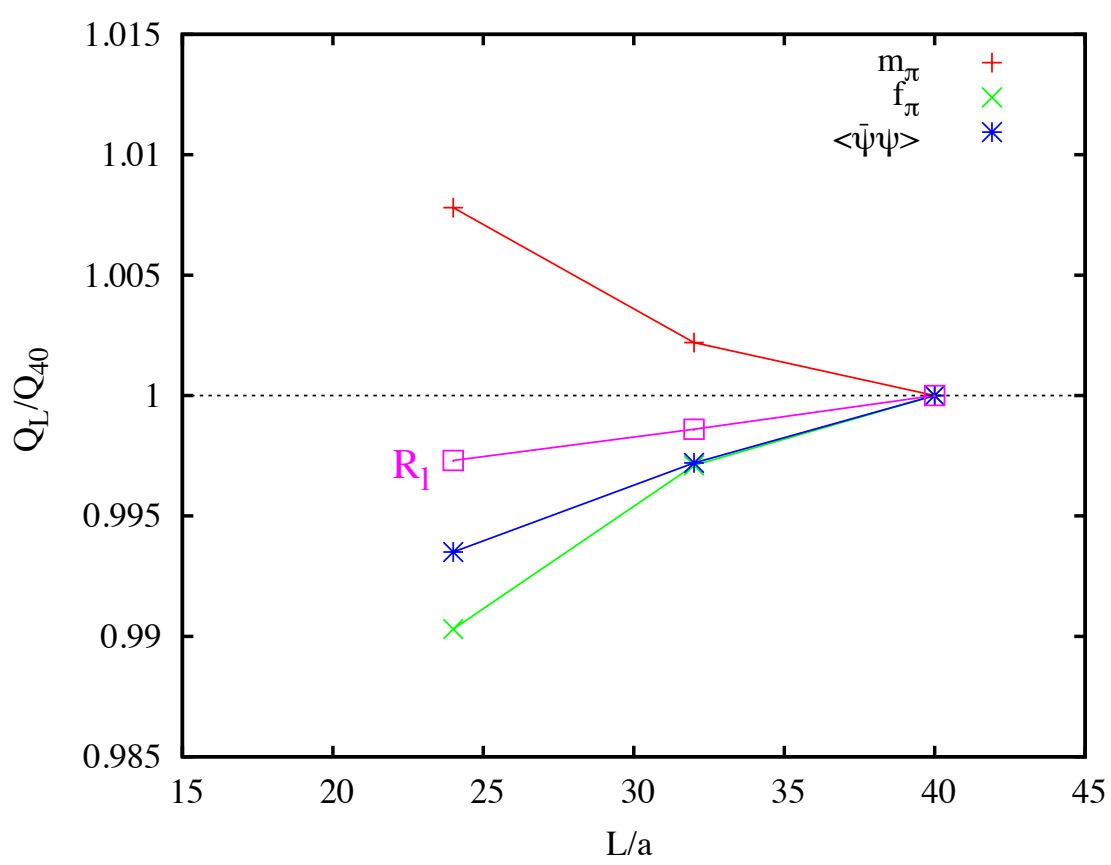

Figure 4: Finite volume effects in different quantities are illustrated by plotting the ratio of the quantity on lattices of spatial length, $L / a$, of 24 and 32 to that on lattices of spatial length 40 . The lattices have the sea quark mass parameters of coarse set 5 . The quantities shown are the pion mass (red pluses), pion decay constant (green crosses) and unsubtracted light quark condensate (blue bursts). Pink squares give the result for the quantity $R_{l}$ defined in Eq. (4.2). Statistical errors (not shown) are approximately $0.1 \%$.

for light quarks and

$$
R_{s}=-\frac{4 m_{s}\left\langle\bar{\psi} \psi_{s}\right\rangle}{\left(f_{\eta_{s}}^{2} M_{\eta_{s}}^{2}\right)}
$$

for strange quarks, where $\eta_{s}$ is the strange-strange fictitious pseudo-scalar meson. The $R_{l}$ and $R_{s}$ have reduced errors from mass tuning and finite size volumes. For example in figure 4 shows a finite volume test at $\beta=6.0, a=0.12 \mathrm{fm}, m_{\pi}=220 \mathrm{MeV}$. The main analysis used $L=32$.

Figure 5 shows clearly the presence of a quadratic divergence with $a^{-2}$ in the raw results for $R_{q}$. This is very 'clean' in the calculations because the form of the divergence is very constrained. Only a term of the form $m_{q} / a^{2}$ is allowed in $\langle\bar{\psi} \psi\rangle$ for staggered quarks, i.e. no term of the form $m_{q}^{2} / a$ can appear. In the ratio $R_{q}$ this term takes the form $C m_{q}^{2} / a^{2}$ where $C$ depends on the meson mass and decay constant. The HISQ formalism has very small discretisation errors, as is clear from the decay constant and meson mass results in [25], and so there is little additional $a$-dependence to confuse the analysis of the divergent pieces.

Because the power divergence is so dominant it is tempting to try to fit the unsubtracted results for $R_{S}$ to a very simple form: $A+B / a^{2}$. This is in fact possible (it is important to include the error in the inverse lattice spacing when doing this since this is larger than the error in $R_{q}$ ) and 1.02(3)+ $0.725(3) / a^{2}$ is obtained which is the dashed line in lefthand plot of Fig. 5. 1.015(11) $+0.229(5) / a^{2}$ was also obtained for $R_{l}$ with $m_{l}=m_{s} / 5$ and $1.00(1)+0.130(6) / a^{2}$ for $R_{l}$ with $m_{l}=m_{s} / 10$, shown in the next two plots in Fig. 5. These fits are too naive to be useful, because they miss out many important terms. Consequently the value and error of the intercept, $A$, is unreliable for extracting a 

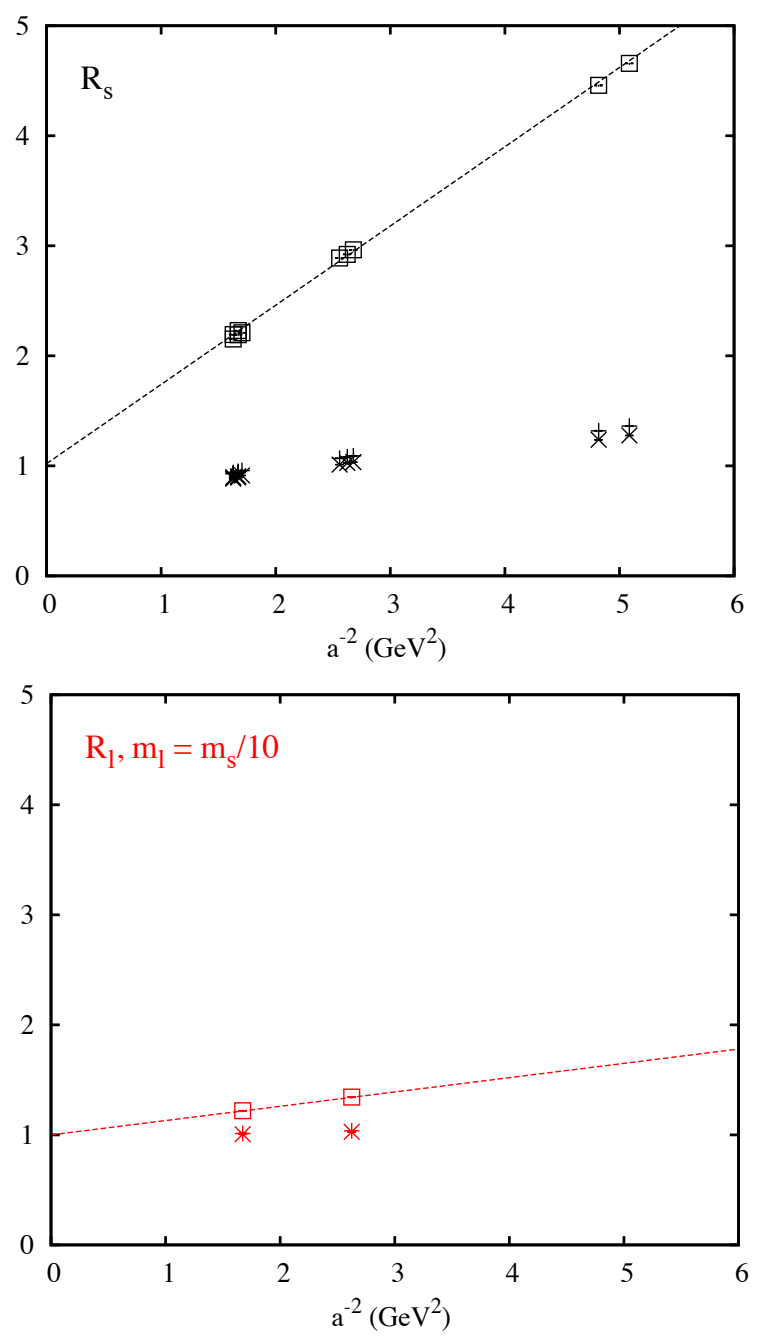

Figure 5: $R_{q}$, defined as the ratio of quark mass times condensate in the $\overline{M S}$ scheme at $2 \mathrm{GeV}$ to the square of the meson mass times decay constant, as a function of the square of the inverse lattice spacing. The top plot shows strange quarks and the bottom plot shows $m_{s} / 10$. The figures shows the result using the condensate without subtraction (squares), tree level subtraction (plus) and one loop subtraction (cross). The value for $\alpha_{s}$ used to multiply the one-loop coefficient was $\alpha_{V}^{n_{f}=4}(2 / a)$. Dashed lines illustrate very simple linear fits to the unsubtracted results as described in the text.

nonperturbative result for $R_{q}$, especially in the $s$ quark case. However, the fits do illustrate that the ratio of slopes is that expected for a term that behaves as $m_{q}^{2} / a^{2}$ (although the simple fit does not allow for the running of the lattice bare quark mass with scale).

One loop perturbation theory is not enough to remove all the divergence, so Bayesian fitting techniques were used, with the following fit models:

$$
R_{q, 0}\left(a, a m_{q}\right)=R_{\mathrm{NP}, \mathrm{phys}}^{(q)}+\delta R_{P T}+\delta R_{a^{2}}+\delta R_{\chi}+\delta R_{\mathrm{vol}}
$$

$R_{q, 0}$ are the lattice results.

$R_{\mathrm{NP}, \text { phys }}$ physical result in the $\overline{M S}$ scheme at $2 \mathrm{GeV}$. 
$\delta R_{\mathrm{vol}}$ finite volume effect $\delta R_{\mathrm{vol}}=v e^{-M L}$

$R_{P T}$ Known tree and one loop results. Also $R_{P T, d i v}=a_{n} \frac{4 \alpha_{s}^{n}\left(a m_{q}\right)^{2}}{\left(a f_{\pi}\right)^{2}\left(a M_{\pi}\right)^{2}}$ and $\delta R_{P T, n o n-d i v}=c_{n} \frac{4 \alpha_{s}^{n}\left(a m_{q}\right)^{4}}{\left(a f_{\pi}\right)^{2}\left(a M_{\pi}\right)^{2}}$. $\delta R_{a^{2}} \delta R_{a^{2}}=\sum_{i=1}^{2} d_{i}\left(\frac{\Lambda a}{\pi}\right)^{2 i}$ with $\Lambda \approx 1 \mathrm{GeV}$.

$\delta R_{\chi}$ includes valence and sea quark mass dependence.

The leading corrections are particularly simple because the chiral logarithms cancel to leave a correction proportional to $M_{\pi}^{2}$. Both $M_{\pi}^{2}$ and $M_{\pi}^{4}$ terms were allowed in the light quark mass fits by defining a chiral expansion parameter

$$
x_{l}=\frac{M_{\pi}^{2}}{2\left(\Lambda_{\chi}\right)^{2}},
$$

and

$$
x_{s}=\frac{\left(M_{\eta_{s}}^{2}-(0.6893(12))^{2}\right)}{2\left(\Lambda_{\chi}\right)^{2}},
$$

with $\Lambda_{\chi}=1.0 \mathrm{GeV}$, and taking

$$
\delta R_{\chi, v a l}=\sum_{i=1}^{2} g_{i}^{(l)} x_{l}^{i}
$$

The $m_{q}=m_{s} / 5, m_{s} / 10$ and $m_{l, p h y s}$ results were fitted with this form taking the prior on the $g_{i}$ coefficients to be $0.0(2.0)$. This allows for a linear term of approximately the size expected in [26]. Higher order terms than $x_{l}^{2}$ have no effect.

The physical results for $R_{q}$ were

$$
\begin{aligned}
R_{l, p h y s} & =-\frac{4 m_{l}\left\langle\bar{\psi} \psi_{l}\right\rangle_{\overline{M S}}(2 \mathrm{GeV})}{\left(f_{\pi}^{2} M_{\pi}^{2}\right)} \\
R_{s, p h y s} & =-\frac{4 m_{s}\left\langle\bar{\psi} \psi_{s}\right\rangle_{\overline{M S}}(2 \mathrm{GeV})}{\left(f_{\eta_{s}}^{2} M_{\eta_{s}}^{2}\right)}
\end{aligned}
$$

The final fits had $\chi^{2} / d o f \approx 0.8$ for 18 dof.

$$
\begin{aligned}
& R_{s, p h y s}=0.574(86) \\
& R_{l, p h y s}=0.985(5) \\
& \frac{R_{s, \text { phys }}}{R_{l, p h y s}}=0.583(84) .
\end{aligned}
$$

The complete error budgets for $R_{s, \text { phys }}, R_{l, \text { phys }}$ and their ratio are given in Table 2 . The substantial $15 \%$ error in $R_{s, \text { phys }}$ reflects the difficulty of extracting a physical result from a power divergent quantity. For $R_{l}$ the error is 17 times better largely because the slope of the divergent piece is 15 times smaller. Errors in $R_{s, p h y s}$ are dominated by errors from the lattice spacing and from fitting the remaining power divergent subtraction terms. There are also substantial errors from statistics and from tuning to the light and strange physical mass points. This is done by tuning the appropriate meson masses through the term $\delta R_{\chi, v a l}$ in Eq. 4.7. This term depends on the lattice spacing through the definition of $x_{l}$ (Eq. 4.5) and $x_{s}$ (Eq. 4.6), because the meson masses appear in $\mathrm{GeV}$ 
Table 2: Error budget for the quantities $R_{s, p h y s}, R_{l, p h y s}$ and their ratio defined in the text. Errors are given as percentages of the final physical result.

\begin{tabular}{llll} 
& $R_{s, \text { phys }}$ & $R_{l, p h y s}$ & $\frac{R_{s, \text { phys }}}{R_{l, p h y s}}$ \\
\hline statistics & 6.1 & 0.2 & 5.1 \\
lattice spacing & 10.0 & 0.3 & 9.7 \\
finite volume & 1.5 & 0.03 & 1.5 \\
$\alpha_{s}$ value & 1.7 & 0.06 & 1.7 \\
fitting power divergence & 7.5 & 0.3 & 7.2 \\
other perturbative subtraction & 1.3 & 0.07 & 1.3 \\
$\chi$ al extrap./interp. $\left(m_{s}\right)$ & 3.0 & 0.1 & 2.9 \\
$\chi$ al extrap./interp. $\left(m_{l}\right)$ & 4.5 & 0.2 & 4.3 \\
$a \rightarrow 0$ extrap. & 1.9 & 0.05 & 1.9 \\
sea mass effects & 0.5 & 0.01 & 0.5 \\
\hline Total & 15 & 0.5 & 14.5
\end{tabular}

units in these terms. The uncertainties in these terms then becomes correlated with the fit to the power divergence, increasing the uncertainty. For $R_{l}$ the power divergence is much less of an issue, but these same terms dominate the final error there as well.

The following values were taken: $m_{s}^{\overline{M S}}(2 \mathrm{GeV})=92.2(1.3) \mathrm{MeV}$ (HPQCD [27]) and $m_{s} / m_{l}=$ 27.41(23) (MILC [28, 29]). These give:

$$
\begin{aligned}
\langle\bar{s} s\rangle^{\overline{M S}}(2 \mathrm{GeV}) & =-0.0245(37)(3) \mathrm{GeV}^{3} \\
& =-(290(15) \mathrm{MeV})^{3} \\
\langle\bar{l} l\rangle^{\overline{M S}}(2 \mathrm{GeV}) & =-0.0227(1)(4) \mathrm{GeV}^{3} \\
& =-(283(2) \mathrm{MeV})^{3}
\end{aligned}
$$

where the second error for each condensate in $\mathrm{GeV}^{3}$ comes from the error in the quark masses.

The ratio of strange to light condensates

$$
\frac{\langle\bar{s} s\rangle^{\overline{M S}}(2 \mathrm{GeV})}{\langle\bar{l} l\rangle^{\overline{M S}}(2 \mathrm{GeV})}=1.08(16)(1),
$$

\subsection{Additional cross-checks}

There were some additional calculations and cross-checks reported in the original paper [18]. The analysis was repeated with gauge configurations generated with ASQTAD sea quarks by the MILC collaboration [28]. The HISQ action was used for the valence correlators. Nine ensembles were used with lattice spacings in the range: $0.15 \mathrm{fm}$ to $0.04 \mathrm{fm}$. the physical result from the fit, which has $\chi^{2} /$ dof $=0.4$ for 20 degrees of freedom. The physical value obtained was

$$
R_{s, \text { phys }}=0.555(84) \text {. }
$$

This is completely consistent with the result from 2+1+1 flavors of HISQ sea quarks in Eq. 4.10, and has a similar error. It is not such a complete calculation, lacking light quark mass results and 
not having such light sea quark masses, and is therefore not our preferred final result. It provides a strong check of our $2+1+1$ result, however, being a completely independent set of numbers. The fits to the $2+1$ results give very similar behaviour to that seen for the $2+1+1$ case, for example choosing a coefficient of the $\alpha_{s}^{2} / a^{2}$ divergence of around 2 .

For the second calculation values of the strange condensate from the HOTQCD collaboration [30] were used. They generated ensembles with an improved gluon action and $u / d$ and $s$ quarks in the sea using the HISQ formalism. Apart from missing $c$ quarks in the sea, the gauge field configurations here are improved through $\mathscr{O}\left(a^{2}\right)$ at tree-level and without tadpole-improvement.

The physical value for $R_{S}$ obtained from the fit was

$$
R_{s}=0.79(34)
$$

This is much less accurate than the result from Eq. 4.10, but agrees both with that and the result from the MILC 2+1 ASQTAD ensembles given earlier in this section. A light quark condensate was not extracted from the HOTQCD results because finite volume sensitivity obscures the power divergence and leads to larger errors.

As an additional cross-check the chiral susceptibility was also computed

$$
\chi_{f}=\frac{\partial}{\partial m_{f}}\left(-\left\langle\bar{\psi} \psi_{f}\right\rangle\right)
$$

The calculation of $\chi_{f}$ has both connected and disconnected contributions, which were computed using noise sources. The results were in reasonable agreement with the values for the condensates at the strange and light quark masses.

\section{Does the chiral condensate exist as a vacuum condensate?}

Brodsky, Shrock and collaborators [31] have suggested that there is no vacuum chiral condensate, but the condensate is associated with a hadron. The GMOR relation is modified so that the condensate term now carries a label for the pion.

$$
\frac{f_{\pi}^{2} M_{\pi}^{2}}{4}=-\frac{m_{u}+m_{d}}{2} \frac{(\langle 0|\bar{u} u+\bar{d} d| 0\rangle)_{\pi}}{2}
$$

There is support for the in medium idea from some Schwinger-Dyson calculations and the light front formalism [32]. See [33] for an extensive discussion of the idea within the Schwinger-Dyson framework.

The contribution of the vacuum QCD condensates to the vacuum energy is a factor of $\sim 10^{45}$ too large. If the QCD vacuum condensates do not exist, then no fine tuning of the condensate is required to remove their contribution.

It would be very difficult to disprove the in medium condensate idea, if the chiral condensate was only estimated from chiral perturbation theory fits to the masses and decay constants of pions and kaons. However, there have been direct lattice QCD calculations of the matrix element in Eq. 2.2, such as the one reviewed here. The chiral condensate has been calculated by many different methods(see [6] for a new technique). There have also been OPE based fits to lattice correlators which have the quark condensate as a parameter [5]. The final results for the chiral condensate are all in good agreement with each other [1], which suggests that lattice QCD calculations support the standard picture of chiral symmetry breaking, with a vacuum chiral condensate. 


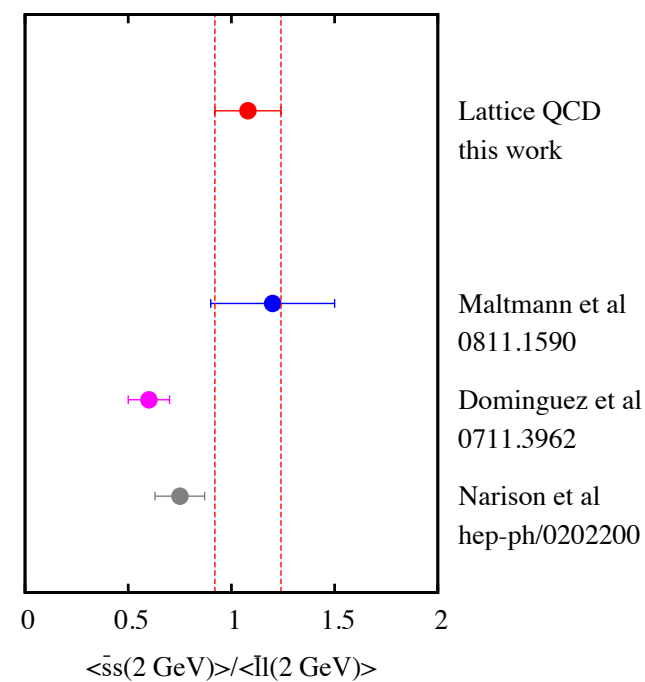

Figure 6: A comparison of results for the ratio of strange to light condensates in the $\overline{M S}$ scheme at $2 \mathrm{GeV}$.

\section{Comparison to sum rule}

In figure 6 the lattice result is compared to results from sum rule calculations [34, 17, 35] for the ratio of strange to light condensates.

Of particular interest to people in the lattice QCD community was the use of decay constant of the $B_{s}$ meson to extract $\langle\bar{s} s\rangle$ using the sum rule method. In 2002 Jamin [26] used $f_{B_{s}} / f_{B}=1.16(4)$ from lattice QCD to estimate $\langle s \bar{s}\rangle_{\overline{M S}} /\langle\bar{l} \bar{l}\rangle_{\overline{M S}}=0.8 \pm 0.3$. In 2008 Maltman [17], updated Jamin's analysis, by using $f_{B_{s}} / f_{B}=1.21(4)$ to estimate $\langle s \bar{s}\rangle_{\overline{M S}} /\langle\bar{l}\rangle_{\overline{M S}} 1.2 \pm 0.3$. The change in values of $f_{B_{s}} / f_{B}$ from lattice QCD was due to the later lattice QCD calculations having light enough pions to see chiral logs. The current average for $f_{B_{s}} / f_{B}$ is 1.202 (22) from lattice QCD, from FLAG 2013 [1].

\subsection{Comparison of $\langle\bar{l} l\rangle^{\overline{M S}}(2 \mathrm{GeV})$ with the chiral condensate}

The value of $\langle\bar{l} l\rangle^{\overline{M S}}(2 \mathrm{GeV})=-(283(2) \mathrm{MeV})^{3}$ can be compared to the value of the chiral condensate. For example the 2013 FLAG review, quotes $\Sigma_{n_{f}=2}=270(7) \mathrm{MeV}$ and $\Sigma_{n_{f}=2+1}=$ 265(17) MeV.

One way to study the mass corrections to the quark condensates is to study the corrections to the Gell-Mann, Oakes, Renner (GMOR) relation. Jamin [26] defined

$$
R_{l}=-\frac{4 m_{l}\left\langle\bar{\psi} \psi_{l}\right\rangle}{\left(f_{\pi}^{2} M_{\pi}^{2}\right)}=1-\delta_{\pi}
$$

Chiral perturbation theory relates the value of $\delta_{\pi}$ to the $H_{2}^{r}$ and $L_{8}^{r}$ Gasser-Leutwyler coefficients.

$$
\delta_{\pi} \propto \frac{M_{\pi}^{2}}{f_{\pi}^{2}}\left(2 L_{8}^{r}-H_{2}^{r}\right)
$$

The new result from lattice QCD is reported in table 3 and compared to two results from sum rules. The errors on $\delta_{\pi}$ were too big to attempt to compute $H_{2}^{r}$. 


\begin{tabular}{c|c|c}
\hline Group & method & $\delta_{\pi}$ \\
\hline This work [18] & lattice & $0.015(5)$ \\
Jamin [26] & sum rule & $0.047(17)$ \\
Bordes et al. [36] & sum rule & $0.06(1)$ \\
\hline
\end{tabular}

Table 3: Comparing values for $\delta_{\pi}$ defined in Eq. 6.1 computed using lattice QCD to results from sum rules.

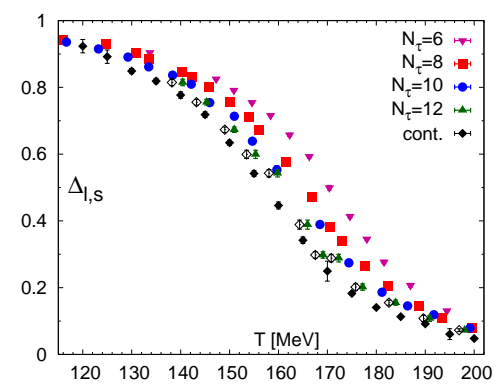

Figure 7: Condensate defined via $\Delta_{l, s}(T)$, as a function of temperature. Figure from [37].

\section{Quark condensates at non-zero temperature}

The quark condensates are important observables for lattice QCD calculations at non-zero temperature. The quark propagator is also used to directly determine the quark condensate at nonzero temperature, so it is interesting to compare the techniques used to remove the divergences, to those used in this calculation.

The HOTQCD collaboration [30, 37], uses the order parameter below at non-zero temperature to determine a quark condensate.

$$
\Delta_{l, s}(T)=\frac{\langle\bar{\psi} \psi\rangle_{l, \tau}-\frac{m_{l}}{m_{s}}\langle\bar{\psi} \psi\rangle_{s, \tau}}{\langle\bar{\psi} \psi\rangle_{l, 0}-\frac{m_{l}}{m_{s}}\langle\bar{\psi} \psi\rangle_{s, 0}} .
$$

The second index on $\langle\bar{\psi} \psi\rangle_{l, \tau}$ is the temperature $\tau$. The perturbative calculation reported in section 3 shows higher order corrections to Eq. 7.1 are small. Figure 7 shows the condensate determined from Eq. 7.1 by Bazavov and Petreczky [37], as a function of the temperature.

There is alternative order parameter for the chiral condensate [14] commonly used at non-zero temperature

$$
\Delta_{q}^{R}=d+2 m_{s} r_{1}^{4}\left(\langle\bar{\psi} \psi\rangle_{q, \tau}-\langle\bar{\psi} \psi\rangle_{q, 0}\right), \quad q=l, s .
$$

where $r_{1}$ is determined from the heavy quark potential [38] and $d$ is a constant. Figure 8 from Bazavov and Petreczky [37], shows the light and strange condensates defined by Eq. 7.2 as a function of temperature.

\section{Conclusions}

There are various possibilities to improve the calculation of the massive quark condensate. For example, it may be possible to compute the next order in perturbation theory for the matching in Eq. 3.1. Some people may want to develop a non-perturbative method to extract the condensates 

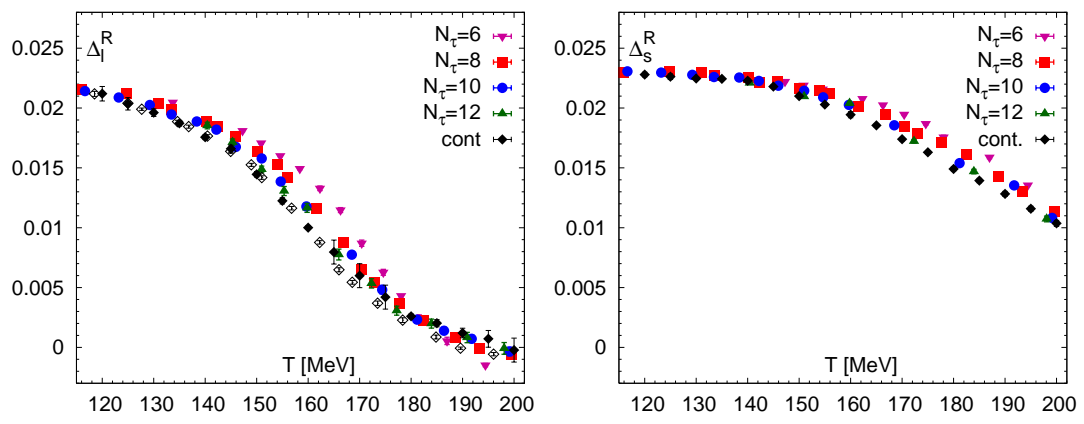

Figure 8: Strange and light condensates (defined from $\Delta_{q}^{R}$ ) as a function of temperature, from [37].

at non-zero quark masses, however this will not be easy, because the matching must include the masses of the quarks.

At this conference Lüscher [20,21] presented a calculation of the quark condensates using the quark flow. The advantage of this method is that it reduces or eliminates the terms, which diverge with powers of the inverse lattice spacing. The value of the condensate from the quark flow has to be converted to the $\overline{M S}$ scheme, so that the value can be compared to that from other approaches.

I have reviewed the first calculation of the mass dependence of the quark condensates, at zero temperature. The summary result for the ratio of the strange condensate to the light condensate is:

$$
\frac{\langle\bar{s} s\rangle^{\overline{M S}}(2 \mathrm{GeV})}{\langle\bar{l} l\rangle^{\overline{M S}}(2 \mathrm{GeV})}=1.08(16)(1)
$$

\section{Acknowledgement}

I thank Bazavov, C. T. H. Davies, R. J. Dowdall, K. Hornbostel, G. P. Lepage, and H. Trottier for an enjoyable collaboration on the work reported here. The calculations described here were performed on the Darwin supercomputer of the Cambridge High Performance Computing service as part of the DiRAC facility jointly funded by STFC, BIS and the Universities of Cambridge and Glasgow. I thank the MILC collaboration for the use of their 2+1+1 gauge field configurations [28].

\section{References}

[1] S. Aoki et al., (2013), arXiv:1310.8555.

[2] L. Reinders, H. Rubinstein, and S. Yazaki, Phys.Rept. 127, 1 (1985).

[3] M. Gell-Mann, R. Oakes, and B. Renner, Phys.Rev. 175, 2195 (1968).

[4] S. Borsanyi et al., Phys.Rev. D88, 014513 (2013), arXiv:1205.0788.

[5] F. Burger, V. Lubicz, M. Muller-Preussker, S. Simula, and C. Urbach, Phys.Rev. D87, 034514 (2013), arXiv: 1210.0838 .

[6] K. Cichy, E. Garcia-Ramos, and K. Jansen, JHEP 1310, 175 (2013), arXiv:1303.1954.

[7] G. Colangelo et al., Eur.Phys.J. C71, 1695 (2011), arXiv:1011.4408.

[8] M. A. Shifman, A. Vainshtein, and V. I. Zakharov, Nucl.Phys. B147, 448 (1979). 
[9] M. A. Shifman, Prog.Theor.Phys.Suppl. 131, 1 (1998), arXiv:hep-ph/9802214.

[10] K. Chetyrkin, V. Spiridonov, and S. Gorishnii, Phys.Lett. B160, 149 (1985).

[11] M. Jamin and M. Munz, Z.Phys. C60, 569 (1993), arXiv:hep-ph/9208201.

[12] E. Shintani et al., Phys.Rev. D82, 074505 (2010), arXiv:1002.0371.

[13] HPQCD Collaboration, J. Koponen et al., PoS LATTICE2010, 231 (2010), arXiv:1011.1208.

[14] Wuppertal-Budapest Collaboration, S. Borsanyi et al., JHEP 1009, 073 (2010), arXiv:1005.3508.

[15] E. Gamiz, M. Jamin, A. Pich, J. Prades, and F. Schwab, JHEP 0301, 060 (2003), arXiv:hep-ph/0212230.

[16] R. Albuquerque, S. Narison, and M. Nielsen, Phys.Lett. B684, 236 (2010), arXiv:0904.3717.

[17] K. Maltman, Phys.Lett. B672, 257 (2009), arXiv:0811.1590.

[18] C. McNeile et al., Phys.Rev. D87, 034503 (2013), arXiv:1211.6577.

[19] HPQCD Collaboration, UKQCD Collaboration, E. Follana et al., Phys.Rev. D75, 054502 (2007), arXiv:hep-lat/0610092.

[20] M. Lüscher, (2013), arXiv:1308.5598.

[21] M. Lüscher, JHEP 1304, 123 (2013), arXiv:1302.5246.

[22] F. David and H. W. Hamber, Nucl.Phys. B248, 381 (1984).

[23] MILC Collaboration, A. Bazavov et al., Phys.Rev. D87, 054505 (2013), arXiv:1212.4768.

[24] G. Kilcup and S. R. Sharpe, Nucl.Phys. B283, 493 (1987).

[25] HPQCD Collaboration, R. Dowdall et al., Phys.Rev. D85, 054509 (2012), arXiv:1110.6887.

[26] M. Jamin, Phys. Lett. B538, 71 (2002), hep-ph/0201174.

[27] C. McNeile, C. Davies, E. Follana, K. Hornbostel, and G. Lepage, Phys.Rev. D82, 034512 (2010), arXiv:1004.4285.

[28] A. Bazavov et al., Rev.Mod.Phys. 82, 1349 (2010), arXiv:0903.3598.

[29] MILC Collaboration, A. Bazavov et al., PoS LAT2009, 079 (2009), arXiv:0910.3618.

[30] A. Bazavov et al., Phys.Rev. D85, 054503 (2012), arXiv:1111.1710.

[31] S. J. Brodsky and R. Shrock, Proc.Nat.Acad.Sci. 108, 45 (2011), arXiv:0905.1151.

[32] S. J. Brodsky, C. D. Roberts, R. Shrock, and P. C. Tandy, Phys.Rev. C85, 065202 (2012), arXiv:1202.2376.

[33] I. C. Cloet and C. D. Roberts, (2013), arXiv:1310.2651.

[34] S. Narison, (2002), hep-ph/0202200.

[35] C. A. Dominguez, N. F. Nasrallah, and K. Schilcher, JHEP 0802, 072 (2008), arXiv:0711.3962.

[36] J. Bordes, C. Dominguez, P. Moodley, J. Penarrocha, and K. Schilcher, JHEP 1005, 064 (2010), arXiv:1003.3358.

[37] A. Bazavov and P. Petreczky, (2013), arXiv:1301.3943.

[38] R. Sommer, Nucl.Phys. B411, 839 (1994), arXiv:hep-lat/9310022. 